\title{
The roles of working memory and intervening task difficulty in determining the benefits of repetition
}

\author{
Dung C. Bui • Geoffrey B. Maddox • David A. Balota
}

Published online: 6 December 2012

(C) Psychonomic Society, Inc. 2012

\begin{abstract}
Memory is better when learning events are spaced, as compared with massed (i.e., the spacing effect). Recent theories posit that retrieval of an item's earlier presentation contributes to the spacing effect, which suggests that individual differences in the ability to retrieve an earlier event may influence the benefit of spaced repetition. The present study examined (1) the difficulty of task demands between repetitions, which should modulate the ability to retrieve the earlier information, and (2) individual differences in working memory in a spaced repetition paradigm. Across two experiments, participants studied a word set twice, each separated by an interval where duration was held constant, and the difficulty of the intervening task was manipulated. After a short retention interval following the second presentation, participants recalled the word set. Those who scored high on working memory measures benefited more from repeated study than did those who scored lower on working memory measures, regardless of task difficulty. Critically, a crossover interaction was observed between working memory and intervening task difficulty: Individuals with low working memory scores benefited more when task difficulty was easy than when it was difficult, but individuals with high working memory scores produced the opposite effect. These results suggest that individual differences in working memory should be considered in optimizing the benefits of repetition learning.
\end{abstract}

Keywords Working memory · Individual differences in memory capacity $\cdot$ Repetition effects $\cdot$ Attention and memory

D. C. Bui $(\bowtie) \cdot$ G. B. Maddox $\cdot$ D. A. Balota

Department of Psychology, Washington University in St. Louis,

St. Louis, MO 63130, USA

e-mail: dcbui@wustl.edu
The spacing effect refers to the finding that spaced repetitions produce better memory performance than massed repetitions. Several accounts of the spacing effect posit that an item's second presentation cues retrieval of the item's first presentation (e.g., Appleton-Knapp, Bjork, \& Wickens, 2005; Benjamin \& Tullis, 2010; Thios \& D'Agostino, 1976). Moreover, when retrieval is successful, it is predicted that the benefit to later memory is positively related to the effort required to successfully retrieve the earlier presentation (e.g., desirable difficulties; Bjork, 1994).

The typical way of examining potential mechanisms underlying the spacing effect is to manipulate the number of items between two study events. Alternatively, one can hold the number of intervening items constant while manipulating the intervening task difficulty (ITD). Using the latter approach, Bjork and Allen (1970) and Roediger and Crowder (1975) presented participants with a set of words two times. Each presentation was separated by an easy or a difficult intervening task. Additionally, each study included control trials that assessed memory immediately following the intervening task (i.e., without a repeated presentation). In both studies, control trial performance was lower following the difficult intervening task than following the easy intervening task, indicating that increasing task difficulty also increased forgetting of the previously presented word set. More important, patterns of recall on the repetition trials differed between the two studies. Bjork and Allen found that memory was better for three-item word sets repeated following the difficult intervening task than following the easy intervening task. In contrast, Roediger and Crowder found that memory was better for 12-item word sets repeated following the easy intervening task than following the difficult intervening task.

Roediger and Crowder (1975) suggested that the differences in materials used across the two studies may have produced the different patterns of results. An additional 
possibility is that there is a trade-off between the difficulty of the intervening task and individual differences. Specifically, if the benefit of repetition relies on the extent to which forgetting occurs during the intervening task, individual differences in episodic memory ability may be important. Indeed, Verkoeijen and Bouwmeester (2008) reported a study in which participants were presented with a list of words, some of which were repeated immediately (a massed condition similar to an easy intervening task, in which minimal forgetting should occur) or after some delay (a spacing condition similar to a difficult intervening task, in which more forgetting should occur). Presentation rate was one word per second (fast rate) or one word per $4 \mathrm{~s}$ (slow rate). Latent class regression analysis on final recall performance revealed two classes: high and low performers. Critically, high performers benefited from spaced repetition over massed repetition regardless of presentation rate, but low performers benefited from spaced repetition only when words were presented at a slow rate. The important point for the present study is that individual differences in overall recall ability appear to modulate the spacing effect, consistent with the argument that retrieval of the earlier event is a contributing mechanism to the spacing effect.

In addition to the role of individual differences in episodic memory, differences in working memory ability may also influence the benefits of repetition. Recently, accumulating evidence has supported a positive correlation between individual differences in working memory and episodic memory (e.g., Kane \& Engle, 2000; McCabe, Roediger, McDaniel, Balota, \& Hambrick, 2010). As McCabe et al. noted, there is considerable evidence that working memory is positively related to attentional processing (e.g., Engle \& Kane, 2004), which in turn plays a role in the recollective processes often required in episodic memory. Consistent with this viewpoint, Oberauer (2005) found a significant correlation between working memory and recollection, but not between working memory and familiarity, suggesting that working and episodic memory may be related because of the attentional control demands placed on each construct.

\section{Present study}

No studies, to our knowledge, have examined whether working memory modulates the benefits of spaced repetition. Given recent theoretical accounts of the spacing effect that suggest that successful retrieval of an item's first presentation on its second presentation is critical to obtaining the benefits of repeated study, it is important to assess the factors that may influence the relative difficulty of such retrieval. Of interest in the present study is evidence suggesting that individuals with higher working memory ability typically show a reduced forgetting rate, relative to low working memory individuals (e.g., Kane \& Engle, 2000). Thus, they should be better at retrieving the first presentation of an item and, hence, show a larger benefit of spacing. Furthermore, individuals with high working memory ability may be more efficient than low-ability individuals at using attentional control to resist interference (Kane \& Engle, 2000), which plays a considerable role in forgetting (e.g., Underwood, 1957). Hence, in the present study, higher working memory ability individuals should be better able to resist interference caused by the intervening task than low working memory ability individuals, which should contribute to a higher likelihood of successful retrieval of the first presentation of the word set.

Importantly, individual differences in working memory may modulate the effect of the difficulty of the intervening task between the repetitions. Specifically, for individuals with low working memory ability, retrieval of the first presentation may be more successful during trials with an easy intervening task, as compared with a more difficult intervening task. In contrast, high working memory ability individuals may possess enough attentional control to resist interference in the difficult intervening condition and, because of the increased desirable difficulties, benefit more from spacing in the difficult intervening condition, as compared with the easy intervening condition.

\section{Experiment 1}

The goal of Experiment 1 was to explore the relationship between working memory and repetition learning in a paradigm that manipulates the difficulty of the intervening material, as in Bjork and Allen (1970) and Roediger and Crowder (1975).

\section{Method}

\section{Participants and design}

Thirty-nine Washington University undergraduate students (17 females; $M$ age $=19.5$ years, $S D=1.5)$ participated for course credit. Both variables (ITD: easy, difficult; trial type: single, repetition) were manipulated within subjects.

\section{Materials}

Word sets Although Bjork and Allen (1970) used sets of three words, pilot data suggested that sets of six items were necessary to avoid ceiling effects. Thus, sets of six words were selected from the English Lexicon Project (Balota et al., 2007) and were controlled for frequency and length. Additionally, words within each set were minimally related, and mean association value for items within a set was 
equated across all stimulus sets (Nelson, McEvoy, \& Schreiber, 1998).

Difficulty manipulation Experiment 1 used the $N$-back task to manipulate the difficulty of the task between repetitions (Kirchner, 1958). In this task, participants are presented single-digit numbers separated by brief visual masks on a computer screen (one per second), and they are told to indicate whether the current number on the screen is the same as or different from the number seen $N$ positions back, using a keypress, with the value of $N$ given at the start of each trial. The purpose of the task is to force participants to maintain and update memory for that $N$ number of items. Difficulty is manipulated by increasing $N$ (the number of items to be held in memory). Pilot data indicated that single trial recall in the one-back $(M=.65, S D=.17)$ and five-back $(M=.57, S D=.20)$ conditions were appropriate manipulations for easy and difficult intervening tasks, respectively.

Working memory measures The letter-number sequencing task and a reading span task were both used to assess working memory. The letter-number sequencing task (adapted from the Wechsler Adult Intelligence Scale, WAIS-IV; Wechsler, 2008) involves participants being shown a series of alternating numbers and letters one at a time at the rate of 1 item per second. Participants are instructed to read each stimulus aloud and to remember them for a subsequent test. At the end of each trial, participants are asked to recall the numbers in numerical order, followed by the letters in alphabetical order. Series length varied between 3 and 12 items, and the entire test consisted of 2 trials of each series length (24 total trials). A trial was considered correct if all stimuli were recalled in the correct order. Total score was calculated by summing the series length of the correct trials. Hence, correct recall of larger series led to a higher score than did smaller series.

The reading span task was adapted from Daneman and Carpenter (1980), with participants being shown a series of alternating sentences and digits. After reading each sentence aloud, participants used a keypress to report whether or not the sentence was sensible, at which time the sentence disappeared and a digit appeared on the screen to be read aloud by the participant. At the end of each series, participants recalled the digits aloud in the order of presentation. Series length varied between two and seven items, and participants completed two trials of each series length. Total score was calculated by summing the series length of the correct trials.

\section{Procedure}

Participants were first administered the letter-number sequencing task and reading span task. Participants then completed the word learning task. On each trial, participants studied a word set for $3 \mathrm{~s}$, which was followed by a $30 \mathrm{~s}$ intervening task. During the intervening task, participants completed an easy or a difficult $N$-back task. Following the intervening task on single-presentation trials, participants were given $20 \mathrm{~s}$ to recall as many words as they could remember from the studied set. Following the intervening task on repetition trials, the same word set from the beginning of the trial was re-presented for $3 \mathrm{~s}$, which was then followed by a $30 \mathrm{~s}$ retention interval (RI) filled with math problems for completion. Following this RI, participants recalled the studied word set.

ITD was blocked, such that participants completed 10 repetition trials and 5 single presentation trials for each level of difficulty, yielding a total of 30 trials. The trials were grouped into cycles that consisted of a repetition trial of each difficulty level and either a difficult or an easy singlepresentation trial. Thus, there were 3 trials per cycle, and ten cycles for the entire word learning task. Within each cycle, the order of the 3 trials was randomized, and the first cycle was administered as a practice cycle.

\section{Results}

Accurate estimates of how task difficulty affects recall required that participants engage in the intervening task. Hence, trials where participants did not respond to at least $80 \%$ of the $\mathrm{N}$ back trials during an intervening task were excluded from analyses, which excluded $3.7 \%$ of the trials. Because performance on the two working memory measures was significantly correlated $(r=.59)$, we created an aggregate working memory score by summing the proportion correct across tasks.

The first question addressed in the present study was whether working memory performance predicted the benefits of repetition learning. We conducted separate hierarchical regressions for the easy and difficult intervening task conditions, with performance on repetition trials as the dependent measure. We first entered performance on the single-presentation trials in the regression model to ensure that any relationship observed between working memory and performance on repetition trials was not simply due to overall differences in episodic memory. Working memory performance was entered in the second step to determine whether it explained any unique variance in repetition recall performance. Table 1 displays the regression coefficients for the easy and difficult intervening task conditions. In both cases, adding working memory to the regression model produced a significant change in $R^{2}, p \mathrm{~s}<.05$. The regression coefficient for working memory in the final model was also significant for both levels of difficulty, $p \mathrm{~s}<.05$.

Next, we examined whether working memory was a stronger predictor of repetition benefits in the difficult intervening task condition than in the easy intervening task condition. If effortful retrieval influences the benefits of spaced 
Table 1 Experiment 1: Hierarchical regression analysis for variables predicting repetition benefits

\begin{tabular}{|c|c|c|c|c|c|c|c|c|}
\hline \multirow[b]{2}{*}{ Variable } & \multicolumn{4}{|c|}{ Easy intervening task } & \multicolumn{4}{|c|}{ Difficult intervening task } \\
\hline & $B$ & $S E B$ & $\beta$ & $\Delta R^{2}$ & $B$ & $S E B$ & $\beta$ & $\Delta R^{2}$ \\
\hline \multicolumn{9}{|l|}{ Step 1} \\
\hline Single-trial recall & .31 & .10 & $.44 *$ & $.20 * *$ & .29 & .14 & $.32 *$ & $.11 *$ \\
\hline \multicolumn{9}{|l|}{ Step 2} \\
\hline Single-trial recall & .18 & .12 & .25 & & .11 & .13 & .12 & \\
\hline Working memory & .19 & .09 & $.35^{*}$ & $.08^{*}$ & .39 & .10 & $.57 * *$ & $.28^{* *}$ \\
\hline
\end{tabular}

repetition, and if there are working memory differences in episodic memory and attentional control, then participants with lower working memory ability should benefit more from repetition trials that follow easy trials, as compared with difficult trials. In contrast, participants with higher working memory should benefit more from spaced repetition learning after a difficult intervening task than after an easy intervening task. In order to address this question, gain scores (i.e., the benefit from repetition learning over single-trial learning) for each ITD condition were computed by the residuals from separate regression analyses that partialed out performance on single-presentation trials from repetition trial performance. Figure 1 displays the regression lines comparing the role of working memory in repetition benefits as a function of ITD. For each participant, we computed the difference between the difficult ITD residual score and easy ITD residual score, which reflected the additional benefit of repetition learning when repetitions were separated by a difficult rather than an easy task. The correlation between these difference scores and

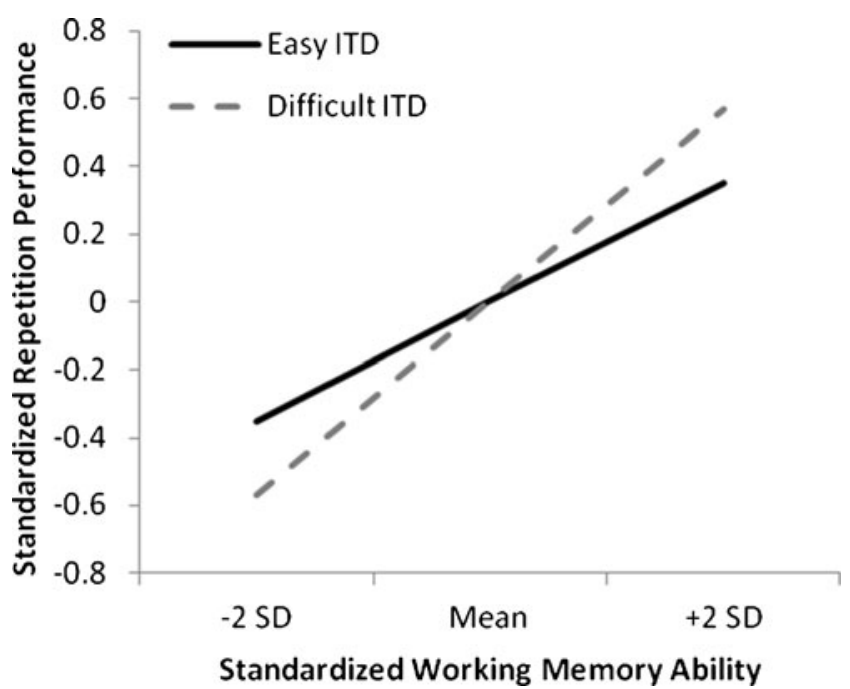

Fig. 1 Predicted standardized repetition performance as a function of intervening task difficulty (ITD) for mean working memory ability and $\pm 2 S D$ in Experiment 1 working memory was significant, $r(37)=.43, p=.007$, indicating that the benefit of separating repetitions by a difficult task, relative to an easy task, generally increases with increased working memory capacity. Thus, although working memory is a predictor of the benefits of repetition in both conditions of ITD, it is a significantly stronger predictor in the difficult intervening task condition.

\section{Discussion}

Experiment 1 yielded two critical findings. First, regression analyses indicated that working memory ability explained unique variance for both levels of ITD even after controlling for single-presentation trial performance (i.e., working memory and the benefits from repetition learning were positively correlated). Second, there was a crossover interaction between working memory ability and ITD such that for low working memory individuals, repetition benefits were greater following an easy intervening task than following a difficult intervening task, whereas the opposite was true for those with high working memory ability.

\section{Experiment 2}

The goal of the second experiment was to replicate the findings from Experiment 1 and extend them to a more diverse sample size, as well as to a different intervening task (consonant-vowel/odd-even switching task; CVOE).

Method

Participants and design

One hundred twelve participants ( 87 females; $M$ age $=$ 31.5 years, $S D=11.0$ ) were recruited from Amazon's Mechanical Turk Web site to take part in this study for monetary compensation. Both variables (ITD: easy, difficult; trial type: single, repetition) were manipulated within subjects. 


\section{Materials}

Working ability was measured using the same tasks as those from the previous experiment (letter-number sequencing and reading span tasks). Stimuli for the word sets were also selected in the same manner as in Experiment 1. Instead of manipulating ITD using an $N$-back task, we used a CVOE switching task.

CVOE task In the CVOE switching task (e.g., Duchek et al., 2009), participants are presented with a letter and a number on each screen and decide whether the letter is a consonant or a vowel or whether the number is odd or even. The type of decision to be made was indicated by an experimenterprovided cue on the screen. On pure block trials, participants made one type of decision (consonant/vowel or odd/ even) throughout the trials. On mixed block trials, the decision to be made switched every 2 number/letter screens. Each trial consisted of 12 number/letter screens, each presented for $2 \mathrm{~s}$. Pilot data confirmed that pure versus mixed trials was a strong manipulation of ITD, with single-trial recall being lower following mixed trials $(M=.55, S D=$ $.17)$ than following the pure trials $(M=.66, S D=.20)$.

\section{Procedure}

The experiment was divided into two sessions. The first session consisted of the two working memory tasks. Following the first session, participants were e-mailed with a link to complete the word learning task. The word learning task was identical to that in Experiment 1, except that during the intervening task, participants completed easy or difficult blocks of the CVOE task (pure or mixed, respectively). For each ITD, participants completed 8 repetition trials and 8 single-presentation trials for each level of difficulty, yielding a total of 32 trials. The trials were grouped into cycles that consisted of a repetition trial of each difficulty level, as well as a single-presentation trial of each difficulty level, yielding 4 trials per cycle, and eight cycles for the entire word learning task. Within each cycle, the order of the 4 trials was randomized, and the first cycle was administered as a practice cycle.
Results

Similar to Experiment 1, trials on which participants did not respond to at least $80 \%$ of the CVOE screens were excluded. In total, $2.4 \%$ of the trials were excluded. Again, the two working memory measures were significantly correlated $(r=$ .37), so we created an aggregate working memory score.

Separate hierarchical regressions were conducted for the easy and difficult intervening task conditions, with performance on repetition trials as the dependent measure. Performance on single-presentation trials was entered in the first step of the regression, followed by working memory performance in the second step. Table 2 displays the regression coefficients for the easy and difficult intervening task conditions. For both difficulty levels, adding working memory to the regression model led to a significant change in $R^{2}, p \mathrm{~s}<.05$. The regression coefficient for working memory in the final model was also significant for both levels of difficulty, $p \mathrm{~s}<.05$.

Our method of examining whether working memory was a stronger predictor of the benefits of repetition as a function of ITD followed the same procedures as those used in Experiment 1. Figure 2 displays the regression lines comparing the role of working memory in repetition benefits as a function of ITD. The correlation between difference scores of the two residuals and working memory was significant, $r(110)=.20, p=.036$. This result replicates the correlation obtained in Experiment 1, which indicated that the benefit of separating repetitions with a difficult task, relative to an easy task, generally increased with increases in working memory capacity. More specifically, working memory was a better predictor of repetition performance (when controlling for single-trial performance) when repetitions were separated by a difficult versus easy task.

\section{General discussion}

The present study examined the relationship between working memory ability and performance in a spaced repetition learning paradigm as a function of ITD. Results from two

Table 2 Experiment 2: Hierarchical regression analysis for variables predicting repetition benefits

\begin{tabular}{|c|c|c|c|c|c|c|c|c|}
\hline \multirow[b]{2}{*}{ Variable } & \multicolumn{4}{|c|}{ Easy intervening task } & \multicolumn{4}{|c|}{ Difficult intervening task } \\
\hline & $B$ & $S E B$ & $\beta$ & $\Delta R^{2}$ & $B$ & $S E B$ & $\beta$ & $\Delta R^{2}$ \\
\hline \multicolumn{9}{|l|}{ Step 1} \\
\hline Single-trial recall & .74 & .04 & $.86^{* *}$ & $.74 * *$ & .69 & .06 & $.76^{* *}$ & $.58 * *$ \\
\hline \multicolumn{9}{|l|}{ Step 2} \\
\hline Single-trial recall & .70 & .05 & $.82 * *$ & & .64 & .06 & $.70 * *$ & \\
\hline Working memory & .12 & .06 & $.11^{*}$ & $.01 *$ & .28 & .07 & $.23 * *$ & $.05^{* *}$ \\
\hline
\end{tabular}




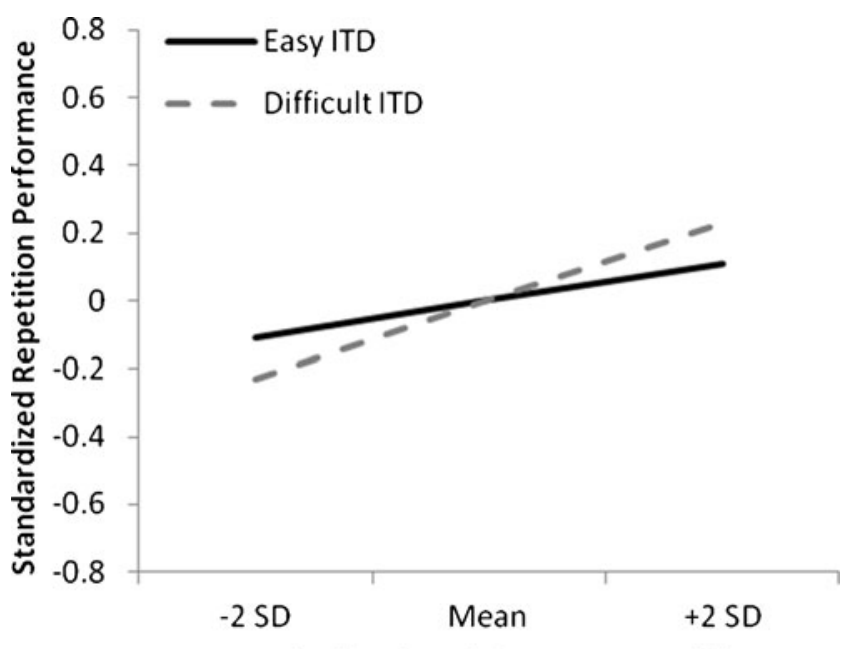

Standardized Working Memory Ability

Fig. 2 Predicted standardized repetition performance as a function of intervening task difficulty (ITD) for mean working memory ability and $\pm 2 S D$ in Experiment 2

experiments revealed a crossover interaction between working memory ability and ITD on the benefits of repetition. As predicted, the interaction reflected a larger benefit of repeated study when events were separated by an easy intervening task rather than a difficult intervening task for low working memory individuals but a larger repetition benefit when presentations were separated by a difficult intervening task rather than an easy intervening task for high working memory individuals.

The crossover interaction observed in the present study suggests that there is a trade-off between memory demands and intervening task demands that influences the effectiveness of repetition learning. If high working memory ability allows for better maintenance of primary task goals through situations of low and high interference, more effortful retrieval should promote greater benefits of repetition in the latter condition. In contrast, for low working memory individuals, successful reminding in high-interference situations may be too difficult (i.e., not desirable), as compared with low-interference situations. This seems to be the case in the present study. In fact, as is shown in Figs. 1 and 2, if an intervening task is very easy, repetition performance may be better for low working memory ability individuals, relative to high working memory ability individuals.

With respect to possible underlying mechanisms of the spacing effect, our results are consistent with accounts that suggest that a stimulus' first presentation is retrieved on its second presentation (e.g., remindings, study-phase retrieval accounts). Of course, the reported regression analyses controlled for differences in episodic memory, which should influence the likelihood of reminding. Thus, one must consider why a more difficult intervening task increases the benefit of repeated study to a greater extent for high working memory individuals. Bjork and Allen (1970) suggested that a more difficult intervening task leads to more variable encoding across study repetitions. Indeed, past aging research suggests that young adults (who tend to have increased working memory ability, relative to older adults) have a higher rate of contextual fluctuation and greater encoding of contextual elements than do older adults (Balota, Duchek, \& Paullin, 1989). Both the study-phase retrieval and encoding variability accounts may contribute to the present pattern of results.

In sum, the present results demonstrate the importance of considering individual differences when investigating the influence of repetition learning. In fact, the different patterns of results observed by Bjork and Allen (1970) and Roediger and Crowder (1975) regarding the influence of the ITD between the repetitions, reviewed in the introduction, may indicate differences in working memory abilities and difficulty of the intervening tasks. Indeed, there appears to be a balance among multiple variables (difficulty of the to-beremembered material, ITD, and individual differences in working memory ability) that modulates the memorial consequences of spaced repetition. In the present study, the conditions that were optimal for high-ability individuals were not optimal for lower ability individuals, thereby highlighting the need to consider the entire range of individual abilities when exploring ways of optimizing learning.

Acknowledgments The authors would like to thank Joel Myerson and Jill Shelton for their feedback and constructive comments about earlier versions of this project, as well as Michael Strube for his help in the analyses and interpretation of the data. This work was supported by NIA Training Grant AG00030 and PO1 AGO3991.

\section{References}

Appleton-Knapp, S. L., Bjork, R. A., \& Wickens, T. D. (2005). Examining the spacing effect in advertising: Encoding variability, retrieval processes, and their interaction. Journal of Consumer Research, 32, 266-276.

Balota, D. A., Duchek, J. M., \& Paullin, R. (1989). Age-related differences in the impact of spacing, lag, and retention interval. Psychology and Aging, 4, 3-9.

Balota, D. A., Yap, M. J., Cortese, M. J., Hutchison, K. A., Kessler, B., Loftis, B.,...Treiman, R. (2007). The English Lexicon Project. Behavior Research Methods, 39, 445-459.

Benjamin, A. S., \& Tullis, J. G. (2010). What makes distributed practice effective? Cognitive Psychology, 61, 228-247.

Bjork, R. A., \& Allen, T. W. (1970). The spacing effect: Consolidation or differential encoding? Journal of Verbal Learning and Verbal Behavior, 9, 567-572.

Bjork, R. A. (1994). Memory and metamemory considerations in the training of human beings. In J. Metcalfe \& A. Shimamura (Eds.), Metacognition: Knowing about knowing (pp. 185-205). Cambridge: MIT Press.

Daneman, M., \& Carpenter, P. A. (1980). Individual differences in working memory and reading. Journal of Verbal Learning and Verbal Behavior, 19, 450-466. 
Duchek, J. M., Balota, D. A., Tse, C. S., Holtzman, D. M., Fagan, A. M., \& Goate, A. M. (2009). The utility of intraindividual variability in selective attention tasks as an early marker for Alzheimer's Disease. Neuropsychology, 23, 746-758.

Engle, R. W., \& Kane, M. J. (2004). Executive attention, working memory capacity, and a two-factor theory of cognitive control. In B. Ross (Ed.), The psychology of learning and motivation (Vol. 44, pp. 145-199). New York: Elsevier.

Kane, M. J., \& Engle, R. W. (2000). WM capacity, proactive interference, and divided attention: Limits on long-term memory retrieval. Journal of Experimental Psychology. Learning, Memory, and Cognition, 26, 336-358.

Kirchner, W. K. (1958). Age differences in short-term retention of rapidly changing information. Journal of Experimental Psychology, 55, 352-358.

McCabe, D. P., Roediger, H. L., McDaniel, M. A., Balota, D. A., \& Hambrick, D. Z. (2010). The relationship between working memory capacity and executive functioning: Evidence for a common executive attention construct. Neuropsychology, 24, 222-243.
Nelson, D. L., McEvoy, C. L., \& Schreiber, T. A. (1998). The University of South Florida word association, rhyme, and word fragment norms. http://www.usf.edu/FreeAssociation/

Oberauer, K. (2005). Control of the contents of working memory - a comparison of two paradigms and two age groups. Journal of Experimental Psychology: Learning, Memory, \& Cognition, 31, 714-728.

Roediger, H. L., \& Crowder, R. G. (1975). The spacing of lists in free recall. Journal of Verbal Learning and Verbal Behavior, 14, 590-602.

Thios, S. J., \& D'Agostino, P. R. (1976). Effects of repetition as a function of study-phase retrieval. Journal of Verbal Learning and Verbal Behavior, 15, 529-536.

Underwood, B. J. (1957). Interference and forgetting. Psychological Review, 64(1), 49-60.

Verkoeijen, P., \& Bouwmeester, S. (2008). Using latent class modeling to detect bimodality in spacing effect data. Journal of Memory and Language, 59, 545-555.

Wechsler, D. (2008). Wechsler Adult Intelligence Scale-Fourth Edition. San Antonio: Pearson Assessment. 